\title{
Impact of minority carrier lifetime on the performance of strained germanium light sources
}

\author{
David S. Sukhdeo ${ }^{1}$, Shashank Gupta ${ }^{1}$, Krishna C. Saraswat ${ }^{1}$, Birendra (Raj) Dutt ${ }^{2,3}$, and \\ Donguk $\mathrm{Nam}^{4} *$ \\ ${ }^{1}$ Department of Electrical Engineering, Stanford University, Stanford, CA 94305, USA \\ ${ }^{2}$ APIC Corporation, Culver City, CA 90230, USA \\ ${ }^{3}$ PhotonIC Corporation, Culver City, CA 90230, USA \\ ${ }^{4}$ Department of Electronic Engineering, Inha University, Incheon 402-751, South Korea \\ E-mail:dwnam@inha.ac.kr
}

\begin{abstract}
We theoretically investigate the impact of the defect-limited carrier lifetime on the performance of germanium (Ge) light sources. For Ge LEDs, we show that improving the material quality can offer even greater enhancements than techniques such as tensile strain, the leading approach for enhancing Ge light emission. For Ge lasers, we show that the defect-limited lifetime becomes increasing important as tensile strain is introduced, and that defect-limited lifetime must be improved if the full benefits of strain are to be realized. We conversely show that improving the material quality supersedes much of the utility of n-type doping for Ge lasers.
\end{abstract}

Keywords

Germanium; Optical Interconnects; Laser; LED; SRH lifetime; Strain 
Germanium (Ge) light sources have garnered much attention for applications in both optical interconnects [1,2] and ultra-compact infrared sensing [3-5] due to Ge's inherent CMOS compatibility. Techniques such as tensile strain and n-type doping have been proposed to enhance the performance of Ge light emitters and extensive modeling exists for these approaches [6-9]. The importance of material quality, however, has been generally overlooked. This is a serious gap in the literature: epitaxial Ge typically suffers from high defect densities and poor carrier lifetimes [10-12], a problem which is well known to inhibit efficient light emission. Moreover, it is important for experimentalists to know whether or not this low carrier lifetime will present a performance bottleneck as Ge light sources mature and, if so, how severe this bottleneck will be. To address these questions, we will theoretically investigate the impact of the defect-limited carrier lifetime on the performance of strained Ge LEDs and lasers. Through our theoretical modeling, we will show that improving the material quality is as important as band engineering in order to achieve efficient Ge LEDs. For Ge lasers, we will show that the defect-limited lifetime is an increasingly critical factor as tensile strain is introduced, and that this defect-limited lifetime must be improved if the full benefits of strain are to be realized. On the other hand, we will show that improving the material quality makes n-type doping a less useful technique for Ge lasers.

Our theoretical modeling process consists of various steps including: full bandstructure calculation, carrier statistics modeling, LED modeling, and laser modeling. The first step is to compute the full bandstructure of strained Ge over the intended range of strain values using $\mathrm{sp}^{3} \mathrm{~d}^{5} \mathrm{~s}^{*}$ tight-binding following the approach of Refs. 13 and 14. As illustrated in Fig. 1 , the use of tight-binding allows us to compute not just the bandstructures' $2 \mathrm{D}$ cross sections (Fig. 1(a)) but also energies over a full 200x200x200 mesh of k-points encompassing the entirety of the first Brillouin Zone (Fig. 1(b)). This gives the full 4D bandstructure, i.e. energy as a function of the three wavevector components $k_{x}, k_{y}$ and $k_{z}$. Using our bandstructure model, we can also study general carrier statistics. To do this, we compute the occupancy probability given by Fermi-Dirac statistics for each allowed k-point and then integrate over the full k-point mesh of allowed energies encompassing the first Brillouin Zone to obtain the carrier concentration in each valley.

Based on the bandstructure model and carrier statistics, it is possible to model the 
performance of a simple Ge LED. For this modeling, we will focus on the internal quantum efficiency (IQE) of a hypothetical Ge LED. Assuming a heterostructure design such that diffusion current is negligible and the injection efficiency will be virtually $100 \%$, which can be achieved even in a simple $\mathrm{Si} / \mathrm{Ge} / \mathrm{Si}$ double heterostructure LED [15], the IQE will simply be the fraction of carrier recombination which is radiative. We can compute the radiative recombination rate using the following equation.

$$
\begin{aligned}
\mathrm{U}_{\text {radiative }} & =\mathrm{R}_{\mathrm{L}} \mathrm{n}_{\mathrm{L}} \mathrm{p}+\mathrm{R}_{\Gamma} \mathrm{n}_{\Gamma} \mathrm{p}=\mathrm{R}_{\mathrm{L}}\left(\mathrm{n}-\mathrm{n}_{\Gamma}\right) \mathrm{p}+\mathrm{R}_{\Gamma} \mathrm{n}_{\Gamma} \mathrm{p} \\
& =\mathrm{R}_{\mathrm{L}} \mathrm{np}+\left(\mathrm{R}_{\Gamma}-\mathrm{R}_{\mathrm{L}}\right) \mathrm{n}_{\Gamma} \mathrm{p}=\mathrm{R}_{\mathrm{L}} \mathrm{np}+\left(\mathrm{R}_{\Gamma}-\mathrm{R}_{\mathrm{L}}\right) \mathrm{np}\left(\frac{\mathrm{n}_{\Gamma}}{\mathrm{n}}\right)
\end{aligned}
$$

$\mathrm{R}_{\mathrm{L}}$ and $\mathrm{R}_{\Gamma}$ are the recombination coefficients for indirect and direct recombination, respectively. $\mathrm{n}_{\mathrm{L}}$ and $\mathrm{n}_{\Gamma}$ are the electron density in the indirect and direct conduction valley, respectively. $\mathrm{n}$ and $\mathrm{p}$ are the total electron and hole density, respectively. $\left(\frac{\mathrm{n}_{\Gamma}}{\mathrm{n}}\right)$ is the fraction of electrons in the direct conduction valley. The non-radiative recombination rate can also be computed using the following equation.

$$
\mathrm{U}_{\text {non-radiative }}=\mathrm{C}_{\mathrm{nnp}} \mathrm{n}\left(\mathrm{np}-\mathrm{n}_{\mathrm{i}}^{2}\right)+\mathrm{C}_{\mathrm{ppn}} \mathrm{p}\left(\mathrm{np}-\mathrm{n}_{\mathrm{i}}^{2}\right)+\mathrm{p} / \tau_{\mathrm{SRH}}
$$

$C_{n n p}$ and $C_{p p n}$ are the recombination coefficients for nnp and ppn Auger process, respectively. $n_{i}$ is the intrinsic carrier density. $\tau_{S R H}$ is the defect-limited carrier lifetime. The radiative recombination rate considers spontaneous emission from both the direct and indirect conduction valleys. Because of the much faster radiative recombination rate of the direct bandgap and also due to the increase of the $\left(\frac{\mathrm{n}_{\Gamma}}{\mathrm{n}}\right)$ term with strain, the total radiative recombination rate can be increased with strain significantly. The non-radiative recombination rate, meanwhile, does not depend on whether electrons are in the direct or indirect conduction valley and is therefore independent of strain. Then, finally, the IQE is simply the ratio of radiative recombination to total recombination as given in the following equation.

$$
\mathrm{IQE}=\frac{\mathrm{U}_{\text {radiative }}}{\mathrm{U}_{\text {radiative }}+\mathrm{U}_{\text {non-radiative }}}
$$

We can consider how the material quality affects the performance of double-heterostructure Ge LEDs, since the defect-limited carrier lifetime, $\tau_{\mathrm{SRH}}$, is a strong function of material quality [16]. In our modeling, we assume that the doping level is $1 \times 10^{19} \mathrm{~cm}^{-3}$. As shown in Fig. 2, there is no conceivable level of strain that will result in an 
efficient Ge LED if $\tau_{\mathrm{SRH}}$ is less than $1 \mathrm{~ns}$. On the other hand, if $\tau_{\mathrm{SRH}}$ can be greater than 10 ns, the IQE can be greater than $50 \%$ for large strain values. Given that epitaxially-grown Ge films tend to have $\tau_{\mathrm{SRH}}$ of approximately $1 \mathrm{~ns}[10,11]$, considerably less than the bulk lifetime values of $>100 \mathrm{~ns}$ for the similar level of n-type doping [17], there is an acute need for research efforts such as those of Refs. 10-12,18 and 19 which explore innovative ways of improving the material quality and thereby improving $\tau_{\mathrm{SRH}}$. Without such efforts, an efficient CMOS-compatible LED is not possible no matter how much research efforts would be put into strain engineering and other techniques.

The penultimate goal of using band-engineered $\mathrm{Ge}$ is to build a low-threshold Ge-on-silicon laser for use in on-chip optical interconnects [20]. Prior modeling has answered some critical questions such as the relative merits of tensile strain and n-type doping [6], the impact of quantum wells [21,22] and the ideal crystal orientation [23], however the importance of material quality for a low-threshold Ge laser has hitherto been mostly overlooked. To finally investigate the importance of material quality, we extend upon our previous theoretical work [6] by singling out $\tau_{\mathrm{SRH}}$ for careful investigation. Our laser modeling in this work employs the same approach explained in detail in our previous work [6] with one exception: we have used a corrected absorption coefficient [24] which better accounts for the splitting of the valence bands under biaxial tensile strain.

In the previous section, we showed that maximizing $\tau_{\mathrm{SRH}}$, presumably through improved material quality [11], is critically important to the performance of a Ge LED. Unsurprisingly, we find that maximizing the $\tau_{\mathrm{SRH}}$ is also very important to achieving a low-threshold $\mathrm{Ge}$ laser. As shown in Fig. 3, there is no combination of strain and doping that gives a useable threshold for the case of a defect-limited carrier lifetime below 1 ns. On the other hand, improving $\tau_{\mathrm{SRH}}$ to $100 \mathrm{~ns}$ makes it possible to achieve thresholds as low as $100 \mathrm{~A} / \mathrm{cm}^{3}$. These benefits appear to start saturating once $\tau_{\mathrm{SRH}}$ reaches about $100 \mathrm{~ns}$. Given that most epitaxial Ge today has a lifetime of only about $1 \mathrm{~ns}$, this means that it is absolutely imperative to improve the material quality if an efficient Ge laser is ever to be realized. Fortunately, this should be within the realm of possibility given that the bulk lifetime of Ge exceeds $100 \mathrm{~ns}$ [17]. Using nanoscale interfacial engineering, the minority carrier lifetime of Ge directly grown on $\mathrm{Si}$ has been already improved up to $9.3 \mathrm{~ns}$ [12]. It is also possible to further improve the minority carrier lifetime of Ge via wafer bonding followed by 
chemical-mechanical polishing [11]. The minority carrier lifetime of $>10$ ns will allow us to achieve a lasing threshold of $<10 \mathrm{kA} / \mathrm{cm}^{3}$ when combined with $\sim 1.9 \%$ biaxial tensile strain [25] and $4 \times 10^{19} \mathrm{~cm}^{-3}$ [26] while it is not possible to achieve such a low lasing threshold for the minority carrier lifetime of $1 \mathrm{~ns}$ regardless of strain and doping.

Due to the strain-induced bandgap narrowing, we observe a large redshift in the emission wavelength with strain as shown in Fig. 4. With 3.0\% strain, the emission wavelength is redshifted from $1550 \mathrm{~nm}$ to nearly $5000 \mathrm{~nm}$. Although the $1550 \mathrm{~nm}$ emission wavelength is important because it corresponds to the C-band minimum loss window for long-distance transmission on erbium-doped fiber, this is not particularly critical for short-distance chip-level optical interconnects since there exist waveguides and short-range optical fibers with reasonable transparency in these wavelength ranges [5]. Moreover, if a Ge light emitter can be strained to redshift its emission wavelength then a Ge modulator or photodetector can also be strained to similarly redshift its operating wavelength, and highly strained $\mathrm{Ge}$ photodetectors with a redshifted detection cutoff have already been demonstrated [27]. More interestingly, the ability to create light sources with the emission wavelength beyond 2000 $\mathrm{nm}$ allows us to create integrated mid-infrared sensors that have recently been attracting more attention [5].

Another interesting observation from our laser modeling is that improving $\tau_{\mathrm{SRH}}$ appears to offer the most benefit for strain and doping combinations that give the smallest threshold. This is shown explicitly in Fig. 5(a) by plotting the threshold versus strain for several different $\tau_{\mathrm{SRH}}$ values, assuming $5 \times 10^{18} \mathrm{~cm}^{-3}$ n-type doping. For strains below $1.0 \%$, there is almost no difference between the curves for $\tau_{\mathrm{SRH}}$ of $10 \mathrm{~ns}$ or greater. At $2.0 \%$ strain, the 10 -ns curve shows a noticeably higher threshold than the 100 -ns and $10-\mu$ s curves, and at $3.0 \%$ strain even the 100 -ns curve has started to diverge from the $1-\mu$ s and $10-\mu$ s curves. The explanation for this phenomenon is that improving $\tau_{\mathrm{SRH}}$ is only helpful when the net carrier lifetime is limited by the defect-assisted recombination process as opposed to other mechanisms such as Auger recombination. In the high threshold regime, the Auger-limited lifetime is about $1 \mathrm{~ns}$ or less, and so it makes little difference whether $\tau_{\mathrm{SRH}}$ is $1 \mu \mathrm{s}$ or $10 \mathrm{~ns}$. In the low-threshold regime, however, the Auger-limited lifetime can be closer to $300 \mathrm{~ns}$. In this case, reducing $\tau_{\mathrm{SRH}}$ from $1 \mu \mathrm{s}$ to $10 \mathrm{~ns}$ will dramatically reduce the net carrier lifetime and thus increase the threshold by more than an order of magnitude. The key takeaway of 
this result is that while material quality is already important to improving the performance of Ge lasers, it will become even more important as techniques such as band engineering and n-type doping start to lower lasing thresholds.

Another important effect of the defect-limited carrier lifetime is that it also affects the optimal doping level: it is known that too much n-type doping can be harmful [6]. From Fig. 3 , we indeed observed that increasing the doping too much will cause the threshold to increase. Also, as can be inferred from plots for various $\tau_{\mathrm{SRH}}$ values in Fig. 3, the optimal amount of n-type doping depends quite strongly on the $\tau_{\mathrm{SRH}}$. For instance, looking at the top of each plot in Fig. 3, we see that for the case of 3.0\% biaxial tensile strain, the threshold is minimized at a doping of more than $1 \times 10^{19} \mathrm{~cm}^{-3}$ when $\tau_{\mathrm{SRH}}$ is $100 \mathrm{ps}$. But for the case of a 1 $\mu \mathrm{s}$ lifetime, we observe that the threshold is minimized for an n-type doping of less than $5 \times 10^{18} \mathrm{~cm}^{-3}$. This "optimal doping," i.e. the doping value which minimizes the threshold, is shown explicitly in Fig. 5(b), wherein we consistently observe that the optimal doping value is lower when $\tau_{\mathrm{SRH}}$ is higher. On a practical level, this result means that n-type doping will become less useful as researchers develop ways to grow Ge with fewer defects and thus longer $\tau_{\mathrm{SRH}}$. As shown in Fig. 5(b), assuming $1.5 \%$ biaxial strain which has recently been realized in Ge-on-Si [28], we find for $\tau_{\mathrm{SRH}}$ of 1 ns that n-type doping will remain useful for concentrations up to $7.2 \times 10^{19} \mathrm{~cm}^{-3}$. However, if improvements in Ge epitaxy can extend $\tau_{\mathrm{SRH}}$ to $100 \mathrm{~ns}$, n-type doping will only be useful at concentrations up to $3.4 \mathrm{x} 10^{19} \mathrm{~cm}^{-3}$ (assuming again $1.5 \%$ strain). Such doping levels are significantly lower than what has already been achieved experimentally [29]. Thus, while very heavy n-type doping may improve a Ge laser's threshold now that $\tau_{\mathrm{SRH}}$ are quite small [10], as the material quality of Ge improves, it will become necessary to actually reduce the n-type doping from present levels in order to achieve the best possible laser performance.

In summary, we show that it is critically important to improve the defect-limited carrier lifetime in order to achieve a high efficiency LED, indicating that much work is needed on improving material quality [11]. Given that state-of-the-art epitaxial Ge has a defect-limited carrier lifetime of only $\sim 1 \mathrm{ns,}$ our modeling suggests that improving this lifetime to $\sim 100 \mathrm{~ns}$ would proportionally improve the efficiency of a Ge LED by two orders of magnitude. This makes lifetime improvements even more critical to LED device performance than band engineering such as biaxial strain. Our modeling also shows that improving the 
defect-limited carrier lifetime is critical for achieving a low-threshold Ge laser. We show that the defect-limited carrier lifetime will become even more important as techniques such as band engineering start to lower lasing thresholds. Interestingly, we observe a negative interaction between material quality and n-type doping: as the material quality improves and reduces the lasing threshold, this will make n-type doping less helpful. This work suggests that to realize a high-efficient Ge LED and a low-threshold Ge laser, we should put more research effort into improving material's quality which has been overlooked so far.

\section{Acknowledgments}

This work was supported by the Office of Naval Research (grant N00421-03-9-0002) through APIC Corporation (Dr. Raj Dutt) and by a Stanford Graduate Fellowship. This work was also supported by an INHA UNIVERSITY Research Grant and by the Pioneer Research Center Program through the National Research Foundation of Korea funded by the Ministry of Science, ICT \& Future Planning (2014M3C1A3052580). The authors thank Boris M. Vulovic for helpful discussions. The authors also thank Ze Yuan for his help implementing the tight-binding code.

\section{References}

[1] K.C. Saraswat, D. Kim, T. Krishnamohan, D. Kuzum, A.K. Okyay, A. Pethe, et al., Germanium for High Performance MOSFETs and Optical Interconnects, ECS Trans. 16 (2008) 3-12.

[2] X. Sun, J. Liu, L.C. Kimerling, J. Michel, Toward a Germanium Laser for Integrated Silicon Photonics, IEEE J. Sel. Top. Quantum Electron. 16 (2010) 124-131.

[3] L. Colace, G. Assanto, Germanium on silicon for near-infrared light sensing, IEEE Photonics J. 1 (2009) 69-79.

[4] V. Sorianello, L. Colace, N. Armani, F. Rossi, C. Ferrari, L. Lazzarini, et al., Low-temperature germanium thin films on silicon, Opt. Mater. Express. 1 (2011) 856.

[5] R. Soref, Mid-infrared photonics in silicon and germanium, Nat. Photonics. 4 (2010) 495-497. 
[6] B. Dutt, D.S. Sukhdeo, D. Nam, B.M. Vulovic, K.C. Saraswat, Roadmap to an efficient germanium-on-silicon laser: strain vs. n-type doping, IEEE Photonics J. 4 (2012) 2002-2009.

[7] G. Pizzi, M. Virgilio, G. Grosso, Tight-binding calculation of optical gain in tensile strained [001]-Ge/SiGe quantum wells., Nanotechnology. 21 (2010) 055202.

[8] G.-E. Chang, S.-W. Chang, S.L. Chuang, Theory for n-type doped, tensile-strained Ge - $\operatorname{Si}(x) \operatorname{Ge}(y) \operatorname{Sn}(1-x-y)$ quantum-well lasers at telecom wavelength, Opt. Express. 17 (2009) 11246-11258.

[9] Y. Cai, Z. Han, X. Wang, R.E. Camacho-Aguilera, L.C. Kimerling, J. Michel, et al., Analysis of threshold current behavior for bulk and quantum-well germanium laser structures, IEEE J. Sel. Top. Quantum Electron. 19 (2013) 1901009.

[10] R. Geiger, J. Frigerio, M.J. Süess, D. Chrastina, G. Isella, R. Spolenak, et al., Excess carrier lifetimes in Ge layers on Si, Appl. Phys. Lett. 104 (2014) 062106.

[11]D. Nam, J.-H. Kang, M.L. Brongersma, K.C. Saraswat, Observation of improved minority carrier lifetimes in high-quality Ge-on-insulator using time-resolved photoluminescence, Opt. Lett. 39 (2014) 6205.

[12] J.J. Sheng, D. Leonhardt, S.M. Han, S.W. Johnston, J.G. Cederberg, M.S. Carroll, Empirical correlation for minority carrier lifetime to defect density profile in germanium on silicon grown by nanoscale interfacial engineering, J. Vac. Sci. Technol. B Microelectron. Nanom. Struct. 31 (2013) 051201.

[13] T. Boykin, G. Klimeck, R. Bowen, F. Oyafuso, Diagonal parameter shifts due to nearest-neighbor displacements in empirical tight-binding theory, Phys. Rev. B. 66 (2002) 125207.

[14] T. Boykin, N. Kharche, G. Klimeck, Brillouin-zone unfolding of perfect supercells having nonequivalent primitive cells illustrated with a $\mathrm{Si} / \mathrm{Ge}$ tight-binding parameterization, Phys. Rev. B. 76 (2007) 035310.

[15] J. Liu, R. Camacho-Aguilera, J.T. Bessette, X. Sun, X. Wang, Y. Cai, et al., Ge-on-Si optoelectronics, Thin Solid Films. 520 (2012) 3354-3360.

[16]E. Gaubas, J. Vanhellemont, E. Simoen, I. Romandic, W. Geens, P. Clauws, Carrier lifetime dependence on doping, metal implants and excitation density in $\mathrm{Ge}$ and $\mathrm{Si}$, Phys. B Condens. Matter. 401-402 (2007) 222-225. 
[17]E. Gaubas, J. Vanhellemont, Dependence of carrier lifetime in germanium on resisitivity and carrier injection level, Appl. Phys. Lett. 89 (2006) 142106.

[18] W.-S. Jung, J.-H. Park, A. Nainani, D. Nam, K.C. Saraswat, Fluorine passivation of vacancy defects in bulk germanium for Ge metal-oxide-semiconductor field-effect transistor application, Appl. Phys. Lett. 101 (2012) 072104.

[19] F. Pezzoli, F. Isa, G. Isella, C.V. Falub, T. Kreiliger, M. Salvalaglio, et al., Ge crystals on Si show their light, Phys. Rev. Applied 1 (2014) 044005.

[20] D. Nam, D.S. Sukhdeo, B.R. Dutt, K.C. Saraswat, (Invited) Light Emission from Highly-strained germanium for on-chip optical interconnects, ECS Trans. 64 (2014) 371-381.

[21] M. Virgilio, M. Bonfanti, D. Chrastina, A. Neels, Polarization-dependent absorption in Ge/SiGe multiple quantum wells: Theory and experiment, Phys. Rev. B. 79 (2009) 075323.

[22] E. Fei, X. Chen, K. Zang, Y. Huo, G. Shambat, G. Miller, et al., Investigation of germanium quantum-well light sources, Opt. Express 23 (2015) 22424.

[23] G.-E. Chang, H.H. Cheng, Optical gain of germanium infrared lasers on different crystal orientations, J. Phys. D. Appl. Phys. 46 (2013) 065103.

[24] J. Liu, L.C. Kimerling, J. Michel, Monolithic Ge-on-Si lasers for large-scale electronic-photonic integration, Semicond. Sci. Technol. 27 (2012) 094006.

[25] A. Gassenq, K. Guilloy, G. Osvaldo Dias, N. Pauc, D. Rouchon, J.-M. Hartmann, et al., $1.9 \%$ bi-axial tensile strain in thick germanium suspended membranes fabricated in optical germanium-on-insulator substrates for laser applications, Appl. Phys. Lett. 107 (2015) 191904.

[26] R.E. Camacho-Aguilera, Y. Cai, N. Patel, J.T. Bessette, M. Romagnoli, L.C. Kimerling, et al., An electrically pumped germanium laser, Opt. Express. 20 (2012) 11316-20.

[27] D. Nam, D. Sukhdeo, A. Roy, K. Balram, S.-L. Cheng, K. C.-Y. Huang, et al., Strained germanium thin film membrane on silicon substrate for optoelectronics, Opt. Express 19 (2011) 25866. 
[28] A. Ghrib, M. El Kurdi, M. Prost, S. Sauvage, X. Checoury, G. Beaudoin, et al., All-around SiN stressor for high and homogeneous tensile strain in germanium microdisk cavities, Adv. Opt. Mater. 3 (2015) 353-358.

[29] Y. Cai, R. Camacho-Aguilera, J.T. Bessette, L.C. Kimerling, J. Michel, High phosphorous doped germanium: Dopant diffusion and modeling, J. Appl. Phys. 112 (2012) 034509. 


\section{Figure Captions}

Fig. 1. The bandstructure of Ge with $2.0 \%$ biaxial tensile strain computed by tight-binding. (a) 2D cross-sectional view. Black dashed horizontal lines are visual aids to help illustrate the $\Gamma$-L energy separation under tensile strain. (b) $3 \mathrm{D}$ cross-sectional view $\left(\mathrm{k}_{\mathrm{z}}=0\right)$. The $\mathrm{x}-$ and y-components of the wavevector (k) are shown, with units given as multiples of the inverse lattice constant (1/a).

Fig. 2. Internal quantum efficiency of a Ge double-heterostructure LED for various $\tau_{\mathrm{SRH}}$ values. Doping is assumed to be $1 \times 10^{19} \mathrm{~cm}^{-3}$. (a) Linear scale in y-axis. (b) Logarithmic scale in y-axis.

Fig. 3. Threshold current density vs. biaxial strain and n-type doping, shown for various $\tau_{\mathrm{SRH}}$ values. In all cases a double heterostructure design with a 300 -nm thick Ge active region and zero optical cavity loss is assumed.

Fig. 4. Emission wavelength vs. biaxial tensile strain and n-type doping. Ge thickness is assumed to be $300 \mathrm{~nm}$ with an optical cavity loss of zero and $\tau_{\mathrm{SRH}}=100 \mathrm{~ns}$.

Fig. 5. (a) Threshold current density vs. biaxial strain assuming a 300-nm thick Ge active region with $5 \times 10^{18} \mathrm{~cm}^{-3}$ n-type doping, shown for various $\tau_{\mathrm{SRH}}$ values. (b) Optimal doping vs. biaxial strain, shown for various $\tau_{\mathrm{SRH}}$ values. In all cases a double heterostructure design with zero optical cavity loss is assumed. 
Figures

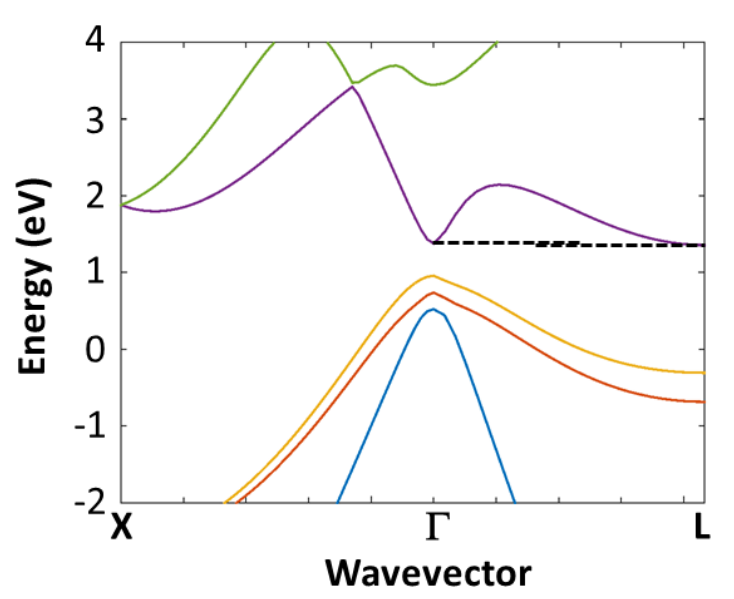

(a)

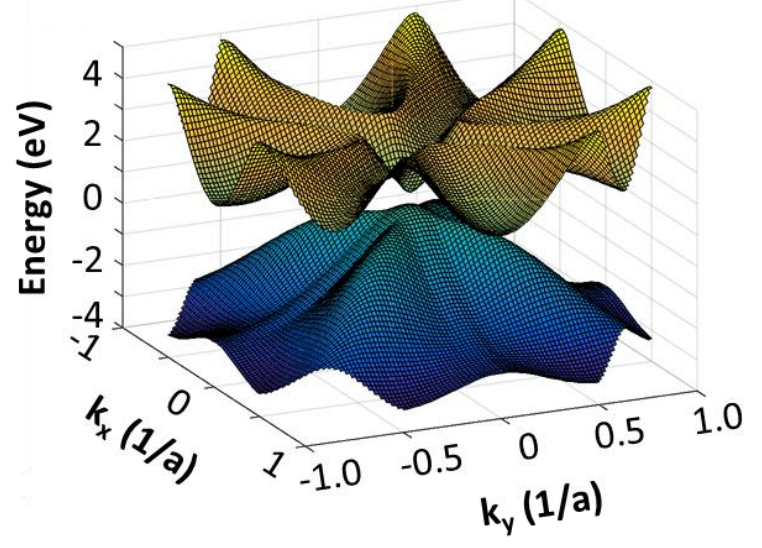

(b)

Fig.1.

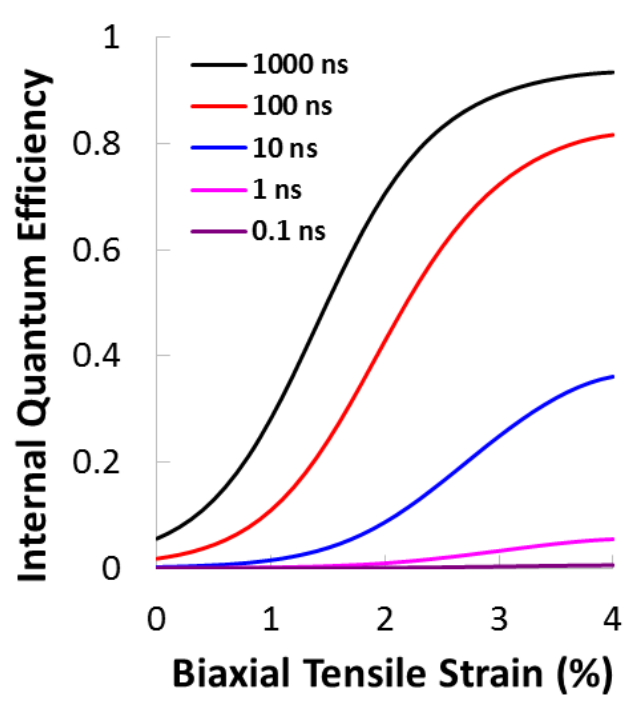

(a)

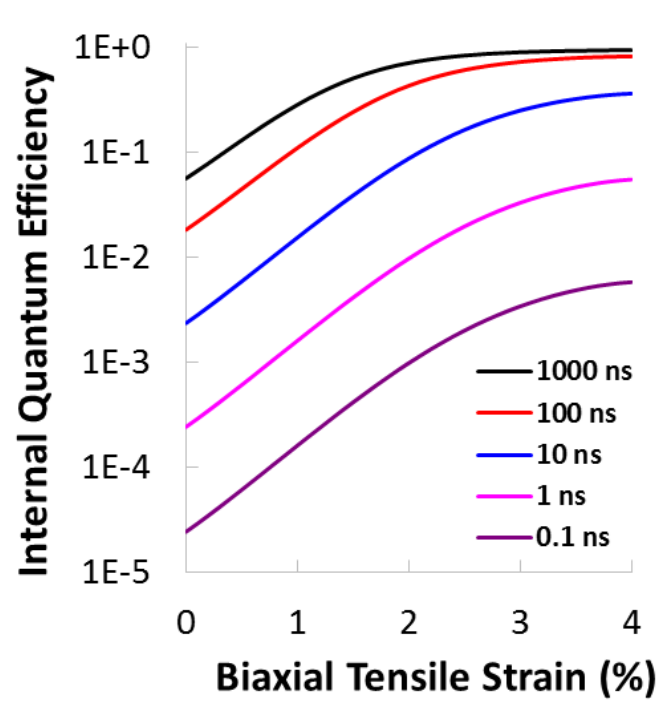

(b)

Fig.2. 

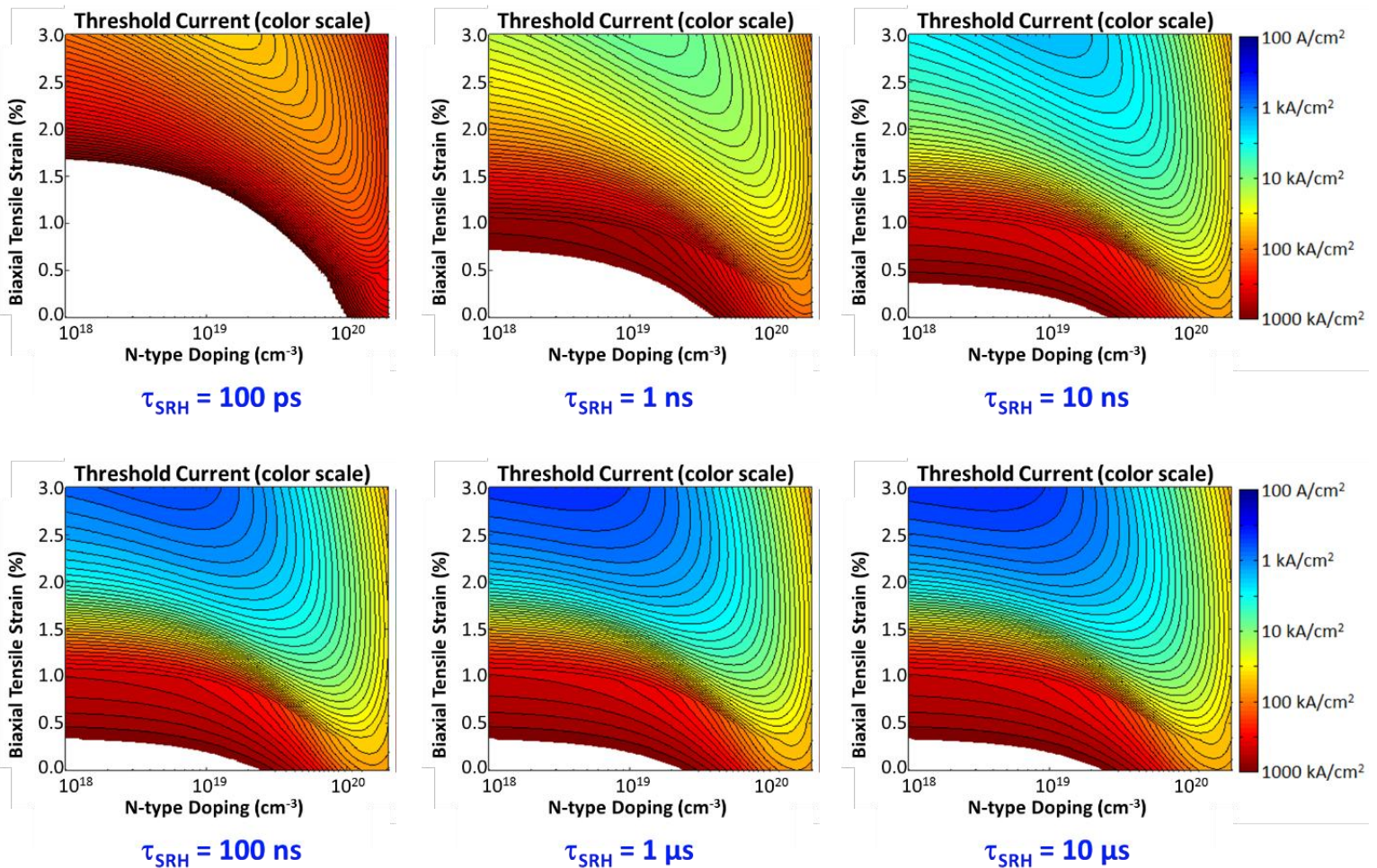

Fig. 3.

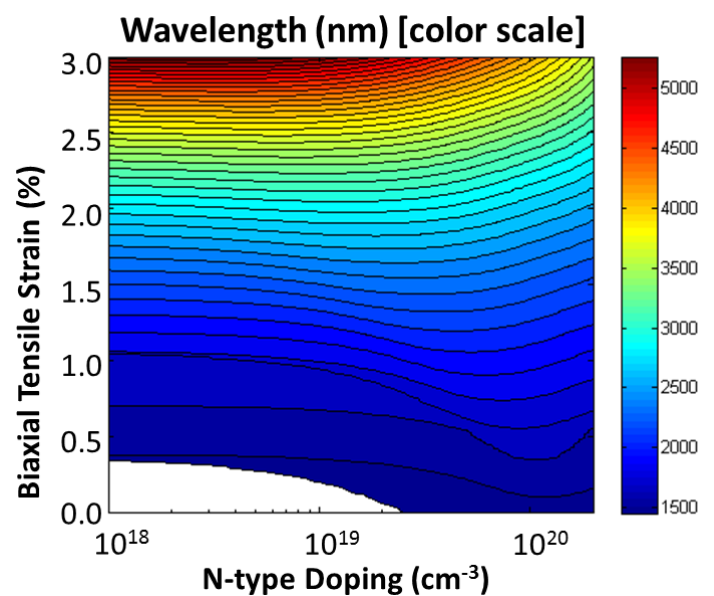

Fig.4. 


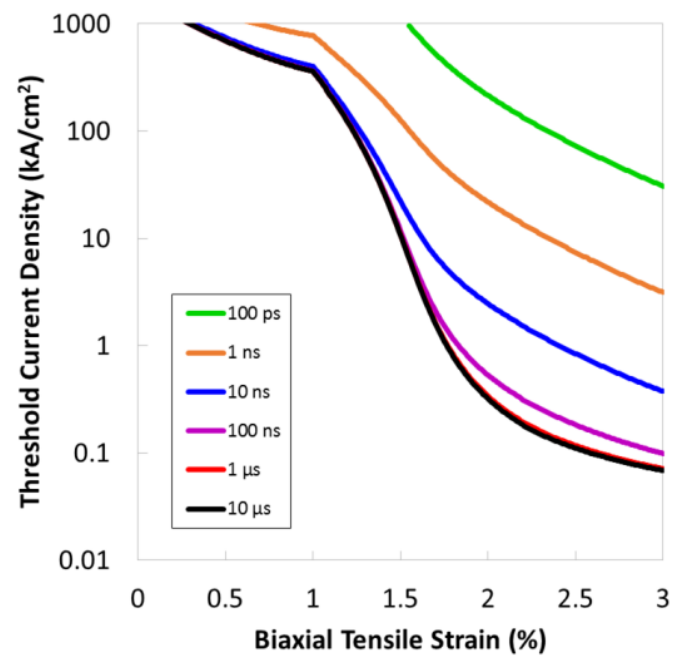

(a)

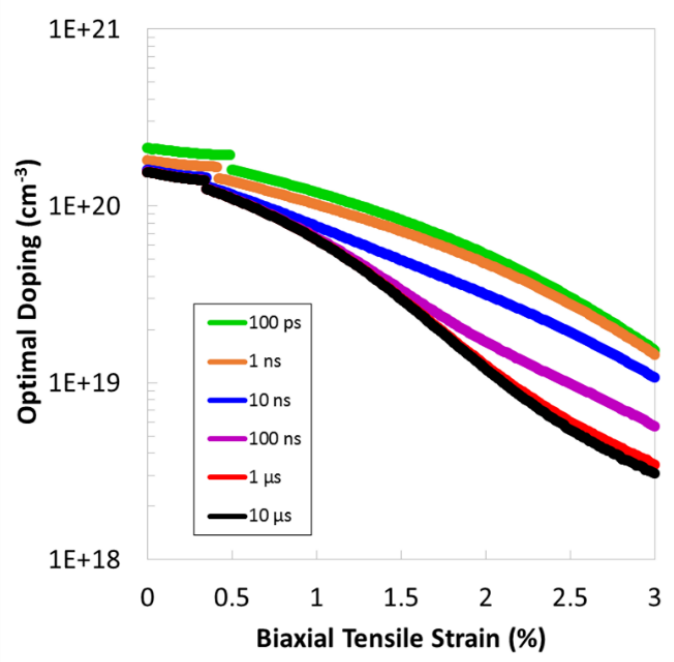

(b)

Fig.5. 